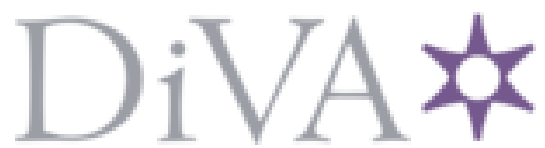

http://www.diva-portal.org

This is the published version of a paper presented at 2019 International Technical Meeting of The Institute of Navigation.

Citation for the original published paper:

Zhang, K. (2019)

On the Effects of Distance-decreasing Attacks on Cryptographically Protected GNSS

Signals

In: Proceedings of the 2019 International Technical Meeting of The Institute of Navigation (pp. 363-372).

N.B. When citing this work, cite the original published paper.

Permanent link to this version:

http://urn.kb.se/resolve?urn=urn:nbn:se:kth:diva-248026 


\section{On the Effects of Distance-decreasing Attacks on Cryptographically Protected GNSS Signals}

\author{
Kewei Zhang \\ Networked Systems Security Group \\ KTH Royal Institute of Technology \\ Stockholm, Sweden \\ kewei@kth.se
}

\author{
Panos Papadimitratos \\ Networked Systems Security Group \\ KTH Royal Institute of Technology \\ RISE SICS \\ Stockholm, Sweden \\ papadim@kth.se
}

\begin{abstract}
The security of global navigation satellite systems draws attention increasingly, and authentication mechanisms for civilian services seem very effective in thwarting malicious behavior. For example, the Galileo E1 Open Service introduces navigation message authentication. Authentication, as well as encryption at navigation message or spreading code level, can prevent spoofing attacks, but do not preclude replay attacks. In this work, we consider a type of strong replay attacks, distance-decreasing attacks, against cryptographically protected GNSS signals. Distance-decreasing attack enhance an attacker's capability of allowing it to mislead the victim receiver that the GNSS signals arrive earlier than true signals. We analyze the instantiation and the effects of the distance-decreasing attacks on unprotected GNSS signals, on navigation message authenticated signals, and on spreading-code encrypted signals. We discuss different strategies that the attacker can adopt to introduce the least bit errors to the re-transmitted signals and avoid being detected at the victim receiver. We provide evaluation results of distance-decreasing attacks on unprotected signals and authenticated navigation message signals, based on different strategies and configurations, and we sketch countermeasures to the different strategies.
\end{abstract}

Index Terms-Distance-Decreasing (DD) attacks, Early Detection (ED), Late Commit (LC), Navigation Message Authentication (NMA), Spreading Code Encryption (SCE)

\section{INTRODUCTION}

Numerous applications require Global Navigation Satellites Systems (GNSS) to provide position or timing services. There is a growing understanding that GNSS-based positioning/navigation can be attacked, thus, the more we rely on smart systems and devices, the higher the stakes are. A lot of research has analyzed vulnerabilities of GNSS-based positioning and attacks, notably jamming, spoofing and replay attacks. Comparing with jamming that blocks the reception of the signals, spoofing and replay are more sophisticated attacks that can forge pseudoranges or navigation messages and consequently mislead the receiver to an arbitrary location. Relatively simple attacks can be mounted with a GNSS signal recorder and an antenna that transmits the recorded signal to the victim, with relatively high power, without any manipulation. More advanced attackers could use GNSS signal simulators to implement a so-called takeover attack seamlessly [10], while considering the targeted victims estimated position and speed: the adversary would transmit a synchronized GNSS signal with low power, then gradually increase the power, to avoid being detected, until the victim would lock on to the spoofed signal. Significant work has been done on devising countermeasures with different perspectives. One approach is to detect the presence of an attacker by checking the received signal strength, e.g., through received power monitoring (RPM) [11], [14] and automatic gain control (AGC) monitoring [1], [2]: the receiver continuously monitors the power of the received signals to check for abnormalities of the signal power. From a different point of view, a properly designed receiver can determine the signal arriving angles with advanced antennas [12], [16]: when adversarial signals are sent from a single device/antenna instead of distinct multiple origins, i.e., satellites, the receiver can detect the attacker by checking angles of the received signals. Moreover, the clock drift can indicate of an attack [11], [13], [14]: monitoring clock variations with an advanced oscillator limits the extent of adversarial manipulation. Other approaches propose countering spoofing attacks with cryptographic methods [4], [8], [19], because the spoofers cannot forge the GNSS signals without knowing the corresponding cryptographic keys. However, this requires upgrading the current satellite systems and receivers hardware; for instance, current civilian GPS receivers do not support cryptographic protection.

Cryptographic methods, very effective in countering spoofing attacks, can be categorized in two classes [7]: those based on authentication and those based on encryption. Authentication-based methods are: navigation message authentication (NMA) and spreading code authentication (SCA). Each NMA message is authenticated with a message authentication code (MAC) embedded in the current navigation messageand validated with a symmetric key disclosed in a later message. The MAC cannot be predicted because the delayed key cannot be predicted either. Galileo plans to implement NMA in its E1B open service [6], [18], while integrating NMA in GPS signals is also under discussion. also under discussion. SCA combines public key cryptography, notably digital signatures created with the corresponding satellite private key, and spread spectrum security codes (SSSC). The SSSC scheme uses pseudorandom bit sequences, inserted into normal spreading code sequences in a fixed time window. SSSC can be 
generated by using the signature embedded in the navigation message as seed. Encryption-based methods also operate at the navigation and the spreading code level. The difference between authentication-based methods and encryption-based methods is that the fronter guarantees origins and integrity of the data or signals and the latter grantees secrecy of the signals and data. The encryption-based methods consists of navigation message encryption and spreading code encryption. Both approaches require symmetric key cryptography to ensure that only authorized receivers benefit from the services.

However, cryptographic protection does not prevent replay attacks, which do not require decrypting or forging messages. Typically, there are three main replay attack types: meaconing [13], spreading code estimation replay (SCER) attacks [8] and distance-decreasing (DD) attacks [20]. Meaconing is relatively limited ability because the attacker records and retransmits signals without any manipulation. SCER attacks estimate the security spreading code based on statistics of the received samples, then reconstruct the signal in real time based on the estimation. Due to the transmission and processing delays introduced by the attacker for either of the two aforementioned attacks, reconstructed signals arrive at the victim receiver with a delay. This results in incrementing pseudorange measurements. Distance-decreasing attacks can eliminate unavoidable processing and transmission delays induced by the attacker. A sophisticated attacker can leverage both classical replay and distance-decreasing attacks to induce positive and negative delays to GNSS signals, in order to force a victim receiver to follow a specifically defined trajectory without manipulating cryptographically protected navigation messages.

This work focuses on the analysis of distance-decreasing attacks on the GNSS signals, considering both unprotected and cryptographically protected signals. Section II provides the background on distance-decreasing attacks and GNSS and Section III analyzes attacks on unprotected GNSS signals and Section IV focuses on the navigation message authenticated signals and spreading-code encrypted signals. Section V evaluates the DD attacks on unprotected signals and NMA signal. Section VI sketches countermeasures before a concluding discussion of future work. .

\section{BACKGROUND}

Distance-decreasing attacks are physical layer attacks which harm secure ranging and distance-bounding protocols, which measure distances based on time-of-flight measurements [5], [15]. DD attacks, mounted in two phases, require two devices: an adversarial receiver (ARX), implementing early detection, and an adversarial transmitter (ATX), implementing late commit, as illustrated in Fig. 1 [20]. The ARX estimates a symbol value based only on its early portion, $T_{E D}$, then conveys the estimated value to the ATX. The ATX constructs a late commit signal with two parts: the first part transmitting a predefined value, independent of the value being estimated by the ARX, for a period $T_{L C}$ (see Fig. 1c), and the second part transmitting the estimated symbol value provided by the ARX, for a period $T_{P S}$. This results in the forged signal arriving at the honest receiver (HRX) $T_{D D}$ earlier than the actual signal would have arrived, as shown in Fig. 1a. $T_{D D}$ is the time gain: $T_{D D}=T_{L C}-T_{E D}$, with the resultant reduction in the measured distance, between the honest transmitter (HTX) and the HRX. The ARX receiver and the ATX transmitter antennas are not necessarily installed at the same place, communicating over a (possibly private) channel (wired or wireless), possibly a fast (high-bit-rate) connection..

GNSS signals: For GPS L1 signals, code division multiple access (CDMA) is used, with different modulation schemes for different signals. L1 C/A and L1 P(Y) code signals use binary phase-shift keying (BPSK); while L1C uses time-multiplexed binary offset carrier (TMBOC) and L1 M-code military signals use binary offset carrier with since phasing $\left(B O C_{s i n}\right)$. Galileo also has different signal plans for different services: E1 Open Service (OS) signals using CDMA with composite binary offset carrier (CBOC) modulation, E6 commercial service (CS) signals using CDMA with BPSK, and E5 signals using Alternative BOC modulation (AltBOC). Similarly, BeiDou $\mathrm{B} 1 \mathrm{I} / \mathrm{Q}, \mathrm{B} 2 \mathrm{I} / \mathrm{Q}$ and B3I/Q signals use BPSK, while BOC is used for B1A signals, MBOC is for B1C signals and AltBOC is used for $\mathrm{B} 2 \mathrm{~A} / \mathrm{B}$ signals. GLONASS employs frequency division multiple access (FDMA) with BPSK modulation combined with direct-sequence spread spectrum (DSSS); started testing CDMA signals on their newly launched satellites since 2011. The basic format of the navigation message for GNSS signals, can be illustrated in Fig. 2. The data rate of GPS L1 C/A signals is $50 \mathrm{bps}$, and each sub-frame has a preamble code, used for synchronization. The data rate of the navigation message transmitted on Galileo E1B channel is $125 \mathrm{bps}$, and each page has 10 bits of preamble code for receiver to synchronize with the signals.

\section{DistancE-DECREASING ATTACKS ON NON-PROTECTED SIGNALS}

The first task for the adversary is to synchronize with satellite signals so that the ARX can figure out the starting point of each bit. This does not require the ARX to compute its position. The signals arriving at the ARX can be written as:

$$
y(t)=\sum_{k} C_{k}(t) D_{k}(t) \cos \left(2 \pi\left(f+f_{d}^{k}\right) t+\theta_{k}\right)+n(t),
$$

where $C(t)$ is the spreading code, $D(t)$ is the message, $f_{d}$ is the Doppler frequency, $\theta$ is the initial carrier phase and $n$ is noise; $k$ is the index for different satellites.

For public signals, after synchronization, the ARX starts collecting the energy for a period $T_{E D}$ (a fraction of the bit duration). The ARX conducts demodulation based on the results of synchronization: carrier phase, $\hat{\theta}$, and Doppler frequency, $\hat{f}_{d}$. Take GPS L1 C/A signals as an example: for each data bit, the tracking output for one millisecond, i.e., one $\mathrm{CA}$ code period, can be written as:

$$
P(l)=\sum_{i=1}^{N} Y_{l}(i) C(i) \cos \left(2 \pi\left(f+\hat{f}_{d}\right) \frac{i}{f_{s}}+\hat{\theta}_{l}\right),
$$




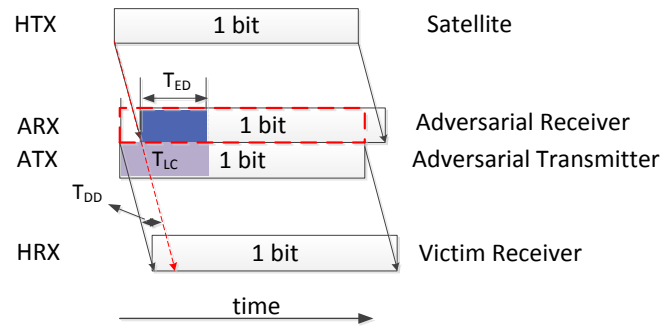

(a) Overview of distance-decreasing attacks

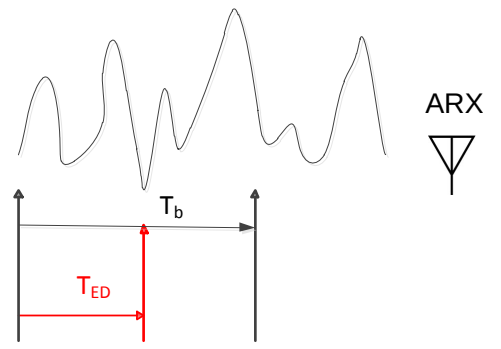

(b) Early-detection at the $A R X$

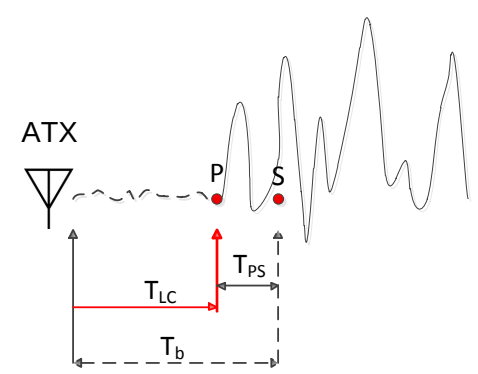

(c) Late-commit at the $A T X$

Fig. 1: Illustration of distance-decreasing attacks

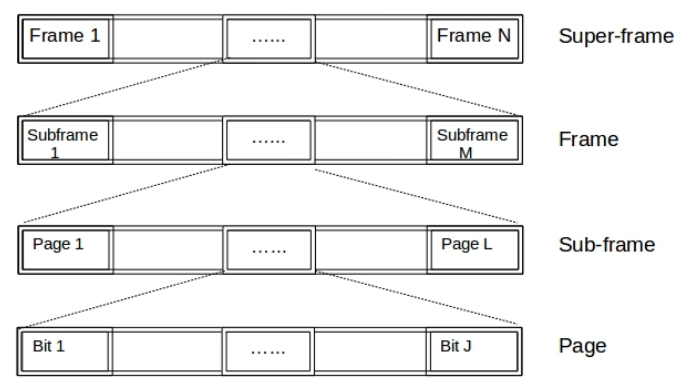

Fig. 2: GNSS navigation message structure

where $P(l)$ is the tracking result corresponding to the $l^{\text {th }} \mathrm{ms}$, $Y_{l}(i)$ is the $i^{t h}$ sample corresponding to the $l^{t h} \mathrm{~ms}$ in one bit, $C(i)$ is the $i^{\text {th }}$ sample of local CA code, $f_{s}$ is sampling frequency and $N$ is number of samples for one CA code. The energy over $T_{E D}$ can be collected, written as:

$$
E_{T_{E D}}=\sum_{l=1}^{T_{E D} * N} P(l) .
$$

The ARX determines the bit value based on the resultant $E_{T_{E D}}$, and it conveys the estimated bit value, $\hat{b}$, together with the carrier phase, $\hat{\theta}$, and Doppler frequency, $\hat{f}_{d}$, to the ATX. The higher $T_{E D}$ is, the closer is the collected energy by the ARX to the actual energy of the data bit.

The ATX already starts transmitting a pre-defined signal, based on some strategy, for a period $T_{L C}$, as shown in Fig. 1c. The reconstructed signal for one bit, at the ATX after receiving the estimated value from the ARX, can be written as:

$S(t)=\left\{\begin{array}{lr}a_{1} \tilde{b}(t) C(t) \cos \left(2 \pi\left(f+\tilde{f}_{d}\right) t+\tilde{\theta}\right) & 0 \leq t<T_{L C} \\ a_{2} \hat{b}(t) C(t) \cos \left(2 \pi\left(f+\hat{f}_{d}\right) t+\hat{\theta}\right) & T_{L C} \leq t \leq T_{b}\end{array}\right.$

where $a_{1}$ and $a_{2}$ are amplitude parameters of the two parts. In absence of other information, the ATX replaces $\left\{\hat{f}_{d}, \hat{\theta}\right\}$ for $\left\{\tilde{f}_{d}, \tilde{\theta}\right\}$ provided by the ARX for the last bit. If the estimated values from the ARX are reliable, $T_{L C}$ should be as short as possible so that the ATX can construct a signal as close to the legitimate one as possible.

The ATX decides to transmit the signal at the beginning of one sub-frame, as there is a known preamble code at the

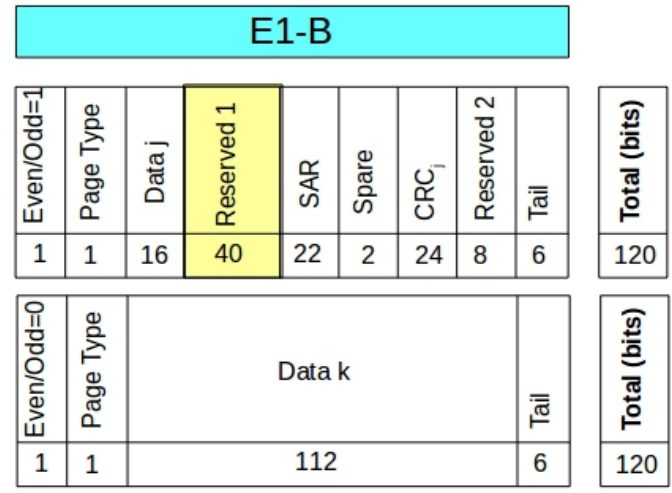

Fig. 3: One nominal page of E1B I/NAV message

beginning of each sub-frame. This can help the attacker to achieve better performance by adjusting $T_{E D}$ and $T_{L C}$. For instance, if the estimated bit value from the ARX is not the same as the preamble bit, the attacker should increase $T_{E D}$ or adopt another estimation mechanism. Meanwhile, $T_{L C}$ should be increased accordingly, to keep the same time gain when $T_{E D}$ increases (recall $T_{D D}=T_{L C}-T_{E D}$ ). If the estimated value is as expected and reliable enough, the attacker can decrease $T_{E D}$ and $T_{L C}$.

\section{Distance-DECREASING ATtaCKS ON CRYPTOGRAPHICALly PROTECTED SIGNALS}

\section{A. Navigation Message Authenticated Signals}

GNSS signals with navigation message authentication contain an unpredictable MAC in each message, used to verify its authenticity and integrity. Take the Galileo OS signals as an illustration: one proposal is to use the 40 bits of 'Reserved 1' field, as presented in Fig. 3 from [17]. Each page, as shown in Fig. 2, consists of a 10-bit long preamble code, 114 bits of information and a 6-bit tail (000000) for a total of 120 bits per page. The I/NAV messages use forward error correction (FEC) with $1 / 2$ convolutional code rate, which encodes an 120 bit page to a 240 -symbol long page, with each encoded page interleaved using a block interleaver with 30 columns and 8 rows. The interleaved symbols are multiplied with the spreading code that is not encrypted, then they are modulated 


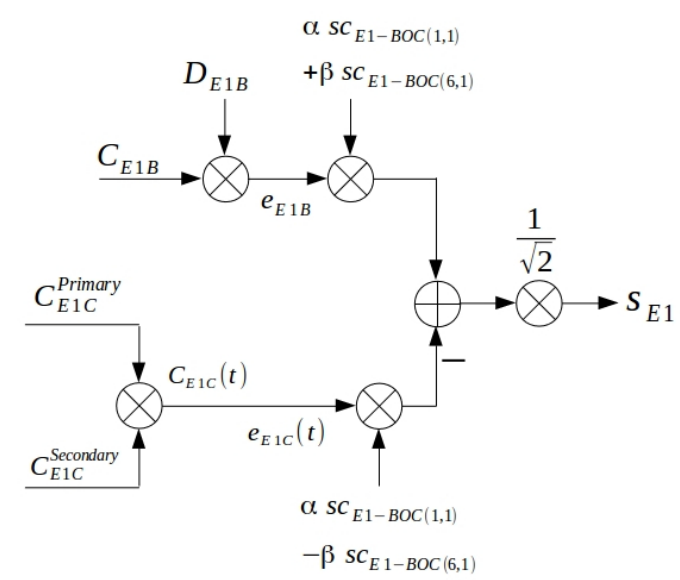

Fig. 4: The Galileo E1 OS signal modulation scheme

on the carrier wave. On the receiver side, the inverse operation recovers the navigation message. The bit rate of the Galileo OS service is $125 \mathrm{bps}$, or $250 \mathrm{symbols} / \mathrm{sec}$, thus the symbol length is $4 \mathrm{~ms}$. The length of the spreading code of the E1B signals is $4 \mathrm{~ms}$, which means that each symbol is multiplied with one spreading code. Recall that the signals arriving at the ARX can be expressed as per Eq. 1. For NMA signals, the MAC part of the $D(t)$ is not predictable.

For the Galileo OS signals, the signals are generated with Composite Binary Offset Carrier $\left(C B O C\left(6,1, \frac{1}{11}\right)\right)$ modulation, with two signal components: $e_{E 1 B}$, the data component on channel $\mathrm{B}$, and $e_{E 1 C}$, the dataless component on channel C, i.e., pilot component, as shown in Fig. 4. The E1 OS composite signal, i.e., $S(t)$, can be expressed as:

$$
\begin{aligned}
& S(t)= \\
& \frac{1}{\sqrt{2}}\left\{e_{E 1 B}(t)\left(\alpha s c_{E 1-B O C(1,1)}(t)+\beta s c_{E 1-B O C(6,1)}(t)\right)\right. \\
& \left.-e_{E 1 C}(t)\left(\alpha s c_{E 1-B O C(1,1)}(t)-\beta s c_{E 1-B O C(6,1)}(t)\right)\right\}
\end{aligned}
$$

where the sub-carrier is $s c_{X}(t)=\operatorname{sgn}\left(\sin \left(2 \pi R_{S, X} t\right)\right)$ and the sub-carrier rate, $R_{S, X}$, is $1.023 \mathrm{MHz}$ for $s c_{E 1-B O C(1,1)}(t)$ and $6.138 \mathrm{MHz}$ for $s c_{E 1-B O C(6,1)}(t)$.

The E1 OS signals arriving at the ARX can be written as:

$$
r(t)=\sum^{k} S\left(t-\tau_{k}\right) \cos \left(2 \pi\left(f+f_{d, k}\right) t+\theta_{k}\right)+n(t)
$$

where $k$ is the satellite number index, $\tau$ is the time delay, $f_{d}$ is the Doppler frequency, $\theta$ is the carrier phase and $n(t)$ is the noise. For Galileo OS signals with NMA, the DD attacker has an advantage: the preamble code of each page is fixed, i.e., 0101100000 , and the spreading codes for different satellites are public. Therefore, after the ARX has locked to the available satellite signals, it can perform early detection for each signal on the subsequent symbols after the preamble code. Each symbol is sampled at baseband frequency as:

$$
\begin{aligned}
S(n)= & d(n) C_{E 1 B}(n)\left[\alpha s c_{E 1 B(1,1)}(n)+\beta s c_{E 1 B(6,1)}(n)\right] \\
& * \cos \left(j 2 \pi f_{d} n+\theta\right) \\
& +C_{E 1 C}(n)\left[\alpha s c_{E 1 B(1,1)}(n)+\beta s c_{E 1 B(6,1)}(n)\right] \\
& * \cos \left(j 2 \pi f_{d} n+\theta\right) \\
& \text { with } n \text { in } 1: f_{S} / r_{d}
\end{aligned}
$$

where $f_{S}$ is the sampling frequency, $r_{d}$ is the data rate, and $d(n) \subset\{+1,-1\}$, for all $\mathrm{n}$. From now on, the simplified notation $s c_{E 1 B(1,1)}(n)$ stands for $s c_{E 1-B O C(1,1)}(n)$ and $s c_{E 1 B(6,1)}(n)$ stands for $s c_{E 1-B O C(6,1)}(n)$. When the ARX tracks the signal continuously, the cosine element can be removed. Then, the signal on E1B channel, after removing the cosine element, becomes:

$$
S(n)=d(n) C_{E 1 B}(n)\left[\alpha s c_{E 1 B(1,1)}(n)+\beta s c_{E 1 B(6,1)}(n)\right]
$$

The symbol value is estimated as follows:

$$
\hat{d}=\sum_{i=1}^{T_{E D} / T_{S}} s(i) * C_{E 1 B}^{\prime}(i)\left[\alpha s c_{E 1 B(1,1)}^{\prime}(n)+\beta s c_{E 1 B(6,1)}^{\prime}(n)\right]
$$

where $C^{\prime}$ and $s c^{\prime}$ are the local replicas of the spreading code and the sub-carriers, and $T_{S}=1 / f_{S}$ is the sampling interval. Eq. 9 implies that the ARX only uses a fraction, i.e., $T_{E D} / T_{d}$, where $T_{d}=4092$ or $4 \mathrm{~ms}$ in duration of a symbol length, of the spreading code to determine the symbol value; in a sense, it can be considered equivalent to $\hat{d}$ modulated with a new spreading code, part of the original one, having the same length as the new symbol $\hat{d}$. Therefore, the new symbol length, $T_{E D}$, and the spreading code has the same length, same frequency and modulation $C B O C(6,1,1 / 11)$. The consequence is that the symbol estimation can and in general will result in higher error rate, because of the shorter spreading code and the shorter integration time. For an individual satellite, the part $C_{E 1 B}(n)\left[\alpha s c_{E 1 B(1,1)}(n)+\beta s c_{E 1 B(6,1)}(n)\right]$ is fixed, therefore the fraction of it, i.e., $C_{E 1 B}^{\prime}(i)\left[\alpha s c_{E 1 B(1,1)}^{\prime}(n)+\beta s c_{E 1 B(6,1)}^{\prime}(n)\right]$, is also fixed, given $T_{E D}$. Hence, the ARX estimates the symbol with a symbol error rate:

$$
P_{s}=\frac{1}{2} \operatorname{erfc}\left(\sqrt{\left(C / N_{0}\right) / R_{s}}\right)
$$

where $R_{s}$ is the new symbol rate, $\frac{1}{T_{E D}}$, and $\operatorname{erfc}(x)=$ $2 / \sqrt{\pi} \int_{t=x}^{\infty} e^{-t^{2}} d t$

When the ARX has acquired the symbol estimated value $\hat{d}$, together with the frequency phase $\hat{\theta}$ and the code delay $\hat{\tau}$, 
it conveys all to the ATX. The newly constructed modulated E1B signal for one symbol at the ATX can be written as:

$$
\begin{aligned}
& S_{A T X}^{B}(t)= \\
& \begin{cases}\tilde{d}(t) c_{B}(t) * \cos \left(2 \pi\left(f+\tilde{f}_{d}\right)(t)+\tilde{\theta}\right) & 0 \leq t<T_{L C} \\
\hat{d}(t) c_{B}(t) * \cos \left(2 \pi\left(f+\hat{f}_{d}\right)(t)+\hat{\theta}\right) & T_{L C} \leq t \leq T_{c}\end{cases}
\end{aligned}
$$

where $c_{B}(t)=C_{E 1 B}(t)\left[\alpha s c_{E 1 B(1,1)}(t)+\beta s c_{E 1 B(6,1)}(t)\right]$. Recall that the Galileo OS signals consist of two channels: B and $\mathrm{C}$; channel $\mathrm{C}$ is used for better acquisition with longer integration time, and channel $\mathrm{B}$ is used for data decoding. Therefore, the ARX can provide two sets of parameters in channel $\mathrm{C}$, one of which is generated at the beginning of each symbol and used for symbol value estimation, while the other one is generated after $T_{E D}$ and conveyed to the ATX. The newly constructed modulated E1C signal at the ATX during the same symbol period can be written as:

$$
\begin{aligned}
& S_{A T X}^{C}(t)= \\
& \begin{cases}c_{C}(t) * \cos \left(2 \pi\left(f+\tilde{f}_{d}\right)(t)+\tilde{\theta}\right) & 0 \leq t<T_{L C} \\
c_{C}(t) * \cos \left(2 \pi\left(f+\hat{f}_{d}\right)(t)+\hat{\theta}\right) & T_{L C} \leq t \leq T_{c}\end{cases}
\end{aligned}
$$

where $c_{C}(t)=C_{E 1 C}(t)\left[\alpha s c_{E 1 B(1,1)}(t)-\beta s c_{E 1 B(6,1)}(t)\right]$.

Hence, the newly constructed E1 OS signals can be written as:

$$
\begin{aligned}
& S_{A T X}(t)= \\
& \left\{\begin{array}{c}
a_{1}\left(\tilde{d}(t) c_{B}(t)-c_{C}(t)\right) * \cos \left(2 \pi\left(f+\tilde{f}_{d}\right)(t)+\tilde{\theta}\right) \\
0 \leq t<T_{L C} \\
a_{2}\left(\hat{d}(t) c_{B}(t)-c_{C}(t)\right) * \cos \left(2 \pi\left(f+\hat{f}_{d}\right)(t)+\hat{\theta}\right) \\
T_{L C} \leq t \leq T_{c}
\end{array}\right.
\end{aligned}
$$

where $a_{1}$ and $a_{2}$ are the amplitude parameters of the two parts of the late commit transmission by the ATX.

\section{B. Spreading Code Encrypted Signals}

Considering encryption of the spreading code, there are the GPS L1 and L2 frequency bands with $\mathrm{P}(\mathrm{Y})$ or $\mathrm{M}$ code, the Galileo channel A signals on the E1 and E6 frequency bands, BeiDou-2 channel Q signals on B1, B2 and B3 bands, and the GLONASS high precision signals. The common features of these signals: their carrier frequencies are disclosed, but the spreading codes are secret, accessed only by the authorized users.

The base-band GNSS signals at the ARX, with an encrypted spreading code, can be written as:

$$
r(t)=a(t) \Psi(t) \cos \left(2 \pi f_{d} t+\theta(t)\right)+N(t)
$$

where $a(t)$ is the amplitude, $\Psi(t)$ is the chip stream that is the result of multiplication of the secret code with the navigation data, and $N(t)$ is the Gaussian noise. The chip length of different encrypted GNSS signals is different. For the Galileo E1 public regulated service (PRS) signals, the code frequency is $2.5575 \mathrm{MHz}$, so the chip length is 391

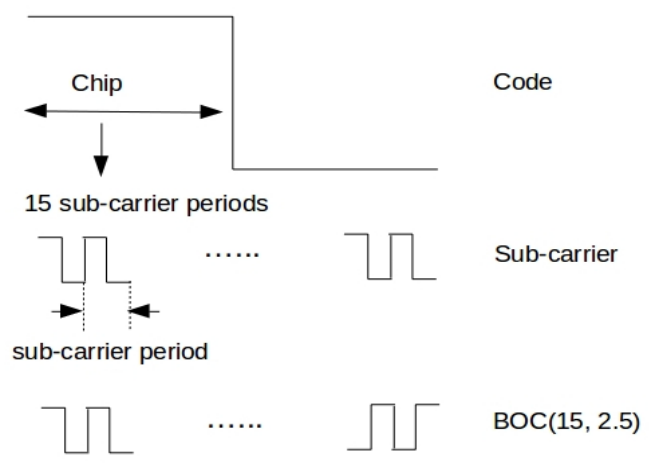

Fig. 5: Illustration of one chip of the Galileo E1 PRS signals

nanoseconds. For the GPS P(Y) code signals, the chip length is 2 microseconds and the length is 195.5 nanoseconds for the GPS M-code signals. More specifically, for GPS M-code signal, with $B O C_{\sin }(10,5)$ modulation, there are 4 repeated rectangular pulses modulated to each chip. For the Galileo E1 PRS signals, with $B O C_{\cos }(15,2.5)$ modulation, there are 12 repeated rectangular pulses per each chip.

The digitalized signal samples at the ARX can be written as:

$$
r(n)=a(n) \Psi(n) \cos \left(2 \pi f_{d} n+\theta(n)\right)+N(n)
$$

where $n$ is the sample index. The first step for the DD attacks is the synchronization with legitimate signals, however, it is hardly possible to do so if the attacker has very little information about the encrypted signals. To do the attacker's favor, it assumes that the attacker can generate code-phase and carrier-phase aligned local replicas, thus the ARX has an good estimation of $f_{d}$ and $\theta$. Assume that the sampling frequency is $f_{S}$, the sampling interval is $T_{S}=1 / f_{S}$, so there are $f_{S} / f_{c}$ samples per chip, where $f_{c}$ is the code frequency. Take the Galileo E1 PRS signals as an illustration, as shown in Fig. 5: after the ARX has aligned with the signals, it starts estimating the chip value based on samples over a period $T_{E D} / T_{S}$. For easier implementation, $T_{E D}$ should be integer multiples of the half of the sub-carrier period. Hence, the ARX first multiplies the next $T_{E D} / T_{S}$ samples with a local cosine signal. The resultant samples over a period $T_{E D}$ are written as:

$$
\hat{\Psi}(l)=r(l) * \cos \left(2 \pi f_{d} l+\theta(l)\right) \quad 1 \leq l \leq T_{E D} / T_{S}
$$

where $l$ is the sample index of the chip. The chip value is estimated by summing all the $T_{E D} / T_{S}$ samples of $\hat{\Psi}$ :

$$
\sum_{l=1}^{T_{E D} / T_{S}} \hat{\Psi}(l) \stackrel{\hat{\Psi}_{m}}{ }=\begin{aligned}
& \gtrless 0 \\
\hat{\Psi}_{m} & =-1
\end{aligned}
$$

where $\hat{\Psi}_{m}$ is the estimation of the $m^{t h}$ chip of $\Psi$. 
Thereafter, $\hat{\Psi}_{m}$, together with the Doppler frequency and the carrier phase, are conveyed to the ATX, which reconstructs the baseband samples of the $m^{\text {th }}$ chip as:

$s_{m}(l)= \begin{cases}a_{1} \tilde{\Psi}_{m}(l) \cos \left(2 \pi \tilde{f}_{d}^{m-1} l+\tilde{\theta}^{m-1}\right) & 1 \leq l<\frac{T_{L C}}{T_{S}} \\ a_{2} \hat{\Psi}_{m}(l) \cos \left(2 \pi \hat{f}_{d}^{m} l+\hat{\theta}^{m}\right) & \frac{T_{L C}}{T_{S}} \leq l \leq \frac{1 / f_{c}}{T_{S}}\end{cases}$

The first part of Eq. 18 is a pre-defined signal and the second part is based on the estimation. In order to keep the parameters consist within one chip, the ATX replaces $\left\{\tilde{f}_{d}^{m}, \tilde{\theta}^{m}\right\}$ for $\left\{\hat{f}_{d}^{m-1}, \hat{\theta}^{m-1}\right\}$, the estimated parameter values of last chip.

\section{Evaluation Results}

\section{A. Simulation Setup}

The distance-decreasing attacker has several receivertransmitter pairs, each targeting one signal. For each such pair, the ARX behaves as a benign receiver before it starts attacking. We assume that the communication between the ARX and ATX is over a low, considered as a negligible delay link.

1) Attacker strategy: The attacker acts at both ends: early detection at the ARX and late commit at the ATX. The early detection is based on the hard decision of an energy detector; the more energy it accumulates for each symbol, the more accurate the symbol estimation is. For distance-decreasing attacks, the newly constructed signal comprises two parts: an attacker-part, in particular, ATX-selectable, followed by the part constructed based on the information provided by the ARX. We consider the following ATX LC approaches for the attacker:

1) Multiply Gaussian noise with the spreading code, a dataless signal.

2) For signals with each symbol multiplied with multiple spreading codes, e.g., GPS L1 C/A signals, transmit $\{+C A$ code, $-C A$ code $\}$ alternately on the carrier wave.

3) Multiply the same symbol value as the one provided by ARX for the immediately preceding with the spreading code.

4) For the spreading-code encrypted signals, transmit the samples of $\tilde{\Psi}(l)$, in Eq. 18, with values of $\{+1,-1\}$ alternatively.

\section{B. Non-protected Signals}

To evaluate the DD attacks on non-protected GNSS signals, we use the recorded raw GPS data of a fixed station at the Aerospace Engineering building of the University of Texas at Austin [9]. The software receiver we used is modified based on [3], which acquires seven satellites at the beginning of the raw data, notably PRN $[23,13,16,3,7,6,19]$. The sky view of the seven available satellites is shown in Fig. 6.

There are three parts in the simulation: first, on the ARX side, receive the authentic GPS signals and start the attack at one bit edge, and then estimate the bit value based on the $T_{E D}$ part of the bit period and conveys the estimation to the second part; second, construct a new signal by combining the ATX

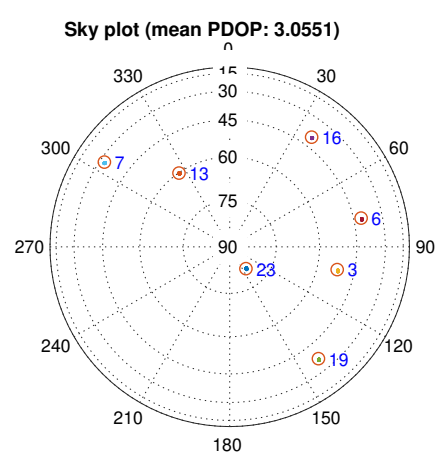

Fig. 6: Sky view of seven satellites

selected signal with the late commit signal based on the ARX input and send the signal to the victim receiver after adding Gaussian noise; third, at the victim receiver side, demodulate the ATX signal, and then compute position using the said signal together with the other available signals. In order to not lose the signal track, the attacker must maintain the carrier phase and the code phase at points $\boldsymbol{P}$ and $\boldsymbol{S}$ shown in Fig. 1c. Moreover, in order to synchronize with the signal, the attacker needs to send the exact preamble code at the beginning of each sub-frame for the GPS L1 C/A signals or at the beginning of each page for the Galileo signals. This is important especially for strategy 2 , because the newly constructed preamble code based on strategy 2 causes loss of synchronization.

We simulate one pair of ARX and ATX to attack one of the seven signals. Fig. 7 shows an example of the effect of the DD attack: the attacker shifts the victim's position 71.9 $\mathrm{km}$ away from its true position by inducing a $1 \mathrm{~ms}$ DD gain on satellite PRN 23, with $T_{L C}=11 \mathrm{~ms}$ and $T_{E D}=10 \mathrm{~ms}$. The resultant computed victim receiver position is the same for each of the aforementioned LC strategies 1, 2, and 3, i.e., $(29.7460792 N,-98.1442799 W)$.

In these simulations, the early detection results are always correct even when $T_{E D}=1 \mathrm{~ms}$ for the raw data we used, with around $50 \mathrm{~dB}-\mathrm{Hz}$ carrier-to-noise ratio [9]. Low $T_{E D}$ is good for the attacker, because given a time gain $T_{D D}=T_{L C}-T_{E D}$, $T_{L C}$ can also be chosen low, resulting in a longer $T_{P S}$ for the ATX to transmit based on the ARX provided information.

As Eq. 4 shows, the power allocated to the two parts of each ATX LC signal bit is $a_{1}$ and $a_{2}$. As the second partis in all likelihood the one that matches the original symbol, the accumulated energy should be dominated by the second part. If $y(t)$ represents the signal after carrier and code removal, the following requirement should be met to have the same result as the ARX estimation:

$$
\int_{0}^{T_{L C}} y(t)<\int_{T_{L C}}^{t_{b}-T_{L C}} y(t)
$$

The left part of Eq. 19 represents the signal power of the first part of Eq. 4, and the right part of Eq. 19 represents the second part. Therefore, for the GPS L1 C/A signals, Eq. 19 can be 


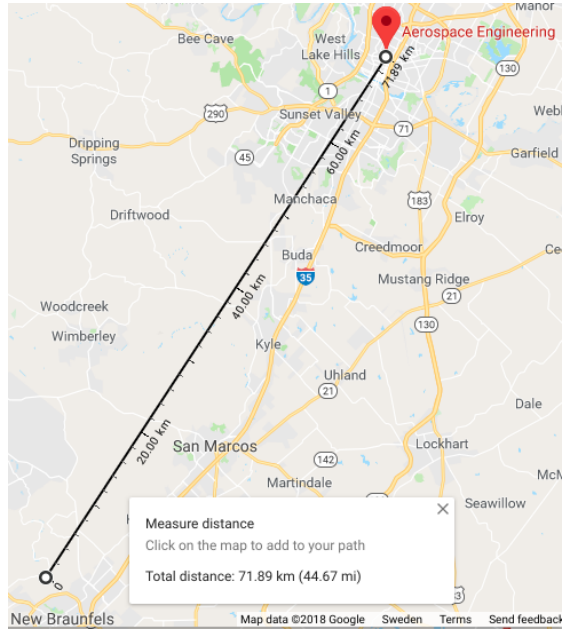

Fig. 7: Distance between the true location and the false location when the attacker introduces $T_{D D}=1 \mathrm{~ms}$ to satellite PRN 23. The map data and the distance measurement are from Google.

written as:

$$
\begin{aligned}
& a_{1} T_{L C}<a_{2}\left(20-T_{L C}\right) \\
& \Longrightarrow \frac{T_{L C}}{20-T_{L C}}<\frac{a_{2}}{a_{1}} \\
& \Longrightarrow y=\frac{T_{L C}}{20-T_{L C}}-\frac{a_{2}}{a_{1}}<0
\end{aligned}
$$

Fig. 8 indicates how to define the amplitude of two LC signal parts to satisfy Eq. 20. Given an amplitude ratio $\frac{a_{2}}{a_{1}}, T_{L C}$ is obtained by Eq. 20. If $T_{L C}$ is a high value, e.g., more than 17 ms, the amplitude ratio must be very high to maintain $y>0$. This could reveal the attack on the victim, the HRX side, based on the significant power increase. $T_{L C}$ should be smaller than $13 \mathrm{~ms}$ with the amplitude ratio smaller than 2 in order to avoid being easily detected by the receiver due to abnormal high power signal. If when $\frac{a_{2}}{a_{1}}>1.2$ is detected as abnormal power jump, then the attacker needs to configure the parameter $T_{L C}<\lfloor 10.9\rfloor$ to pass the detection.

Fig. 9 shows the tracking results of a spoofed signal for period of $100 \mathrm{~ms}$ with different strategies and the corresponding authentic signal. The $\mathrm{x}$-axis is time (in ms) and the $\mathrm{y}$-axis is the prompt code correlation results. $T_{L C}=11 \mathrm{~ms}$ is same for all three approaches, $\frac{a_{2}}{a_{1}}=2$ for approach $1, \frac{a_{2}}{a_{1}}=1$ for approach 2 and $\frac{a_{2}}{a_{1}}=2$ for strategy 3 . The bit values are correct after accumulating all energy of $20 \mathrm{~ms}$ for each strategy. As the figure shows, for strategy 2 , it is not necessary to define a high amplitude ratio because accumulating the energy of period $T_{L C}$ almost equals to zero, due to the alternatively encoding $\{+C A,-C A\}$ with the data bit. We see that there are abnormal jumps in the correlation results that could be used to detect such attacker, if a signal processing level detection mechanism is available to the victim receiver.

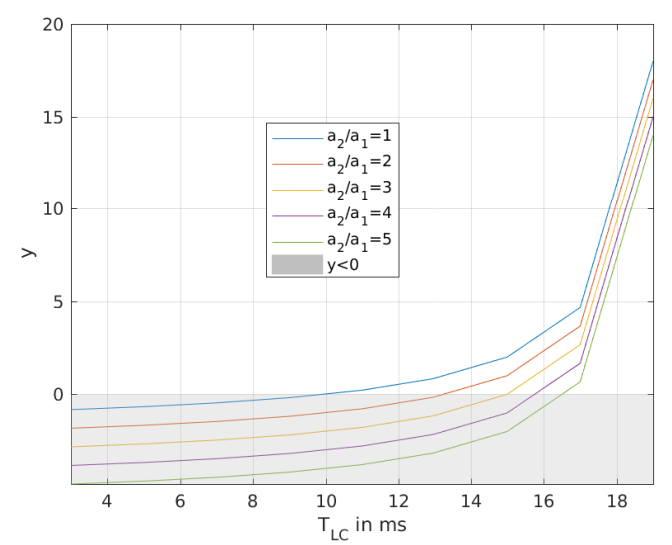

Fig. 8: Relation between $T_{L C}$ and the two parts signal power ratio $\frac{a_{2}}{a_{1}}$ in Eq. 20 .

\section{Navigation Message Authenticated Signals}

In order to evaluate the distance-decreasing attacks on navigation message authenticated GNSS signals, we investigate Galileo E1 OS signals. At the time of this research, we did not have an available Galileo signals at our hands, therefore, we investigated the DD attacks on a simple Matlab simulated signal. As per Section IV, after synchronizing with the authentic signals, the attacker estimates the symbol value based on integration time $T_{E D}$, a multiple of spreading code chips. Fig. 10 shows how $T_{E D}$ affects the symbol estimation based on different signal-noise ratio (SNR) values. We see that the attacker can use lower $T_{E D}$ to estimate the symbol value when $S N R$ is high. Moreover, the figure suggests how to choose the early detection time $T_{E D}$ given an $S N R$, to cope with interference by other PRN codes. For instance, ARX is not able to lock to the satellite for the correct PRN code when $T_{E D}=1023$ chips and SNR is lower than $-20 \mathrm{~dB}$. Therefore, the attacker can design a customized receiver so that the ARX handles a noisy environment and performs well for low $T_{E D}$.

Strategy 2 is not an option for Galileo signals because each symbol is encoded with one spreading code. We consider three symbols $[-1+1-1]$ in the simulation, which are encoded by E1B spreading codes and modulated with the sub-carrier (as shown in Fig 4); we simulate channel B only in this work. The demodulation results are presented in Fig. 11, with $\frac{a_{2}}{a_{1}}=2, T_{L C}=2500$ chips and $S N R=10 \mathrm{~dB}$, assuming that the ARX conveys a correct estimation of the symbol values and the carrier and code phases. We see that the symbol demodulation result is correct after accumulating the energy of all samples of each symbol length. However, there are some jumps in the demodulation results at the transition from the ATX-selected to the ARX-provided part for the LC signal.

The relation between the amplitude ratio $\frac{a_{2}}{a_{1}}$ and $T_{L C}$ for Galileo E1OS signals is similar to Eq. 20, except that $T_{L C}$ is a multiple of chips and the total number of chips for one 

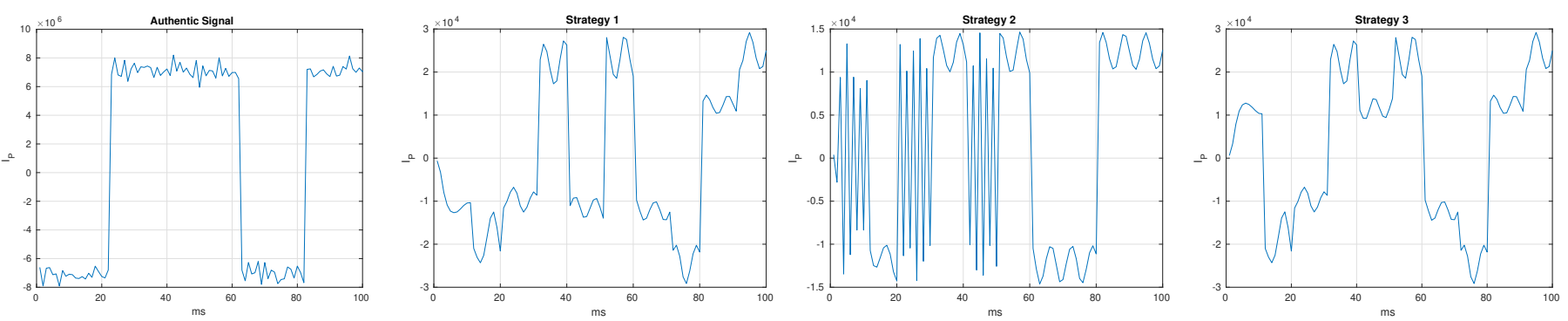

Fig. 9: Prompt code correlation results for authentic signal and spoofed signals based on different strategies
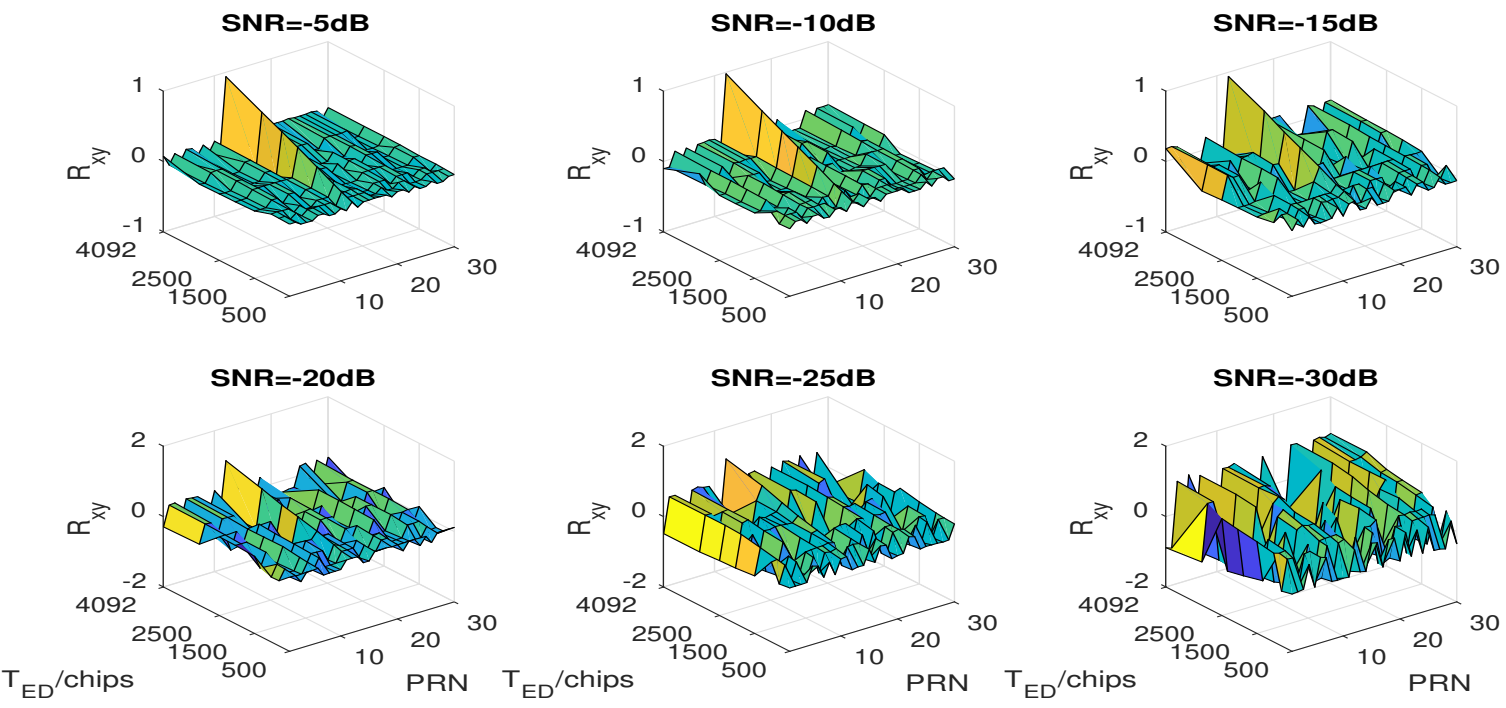

Fig. 10: Spreading codes cross-correlation between PRN 12 and all other PRN codes over period of $T_{E D}$

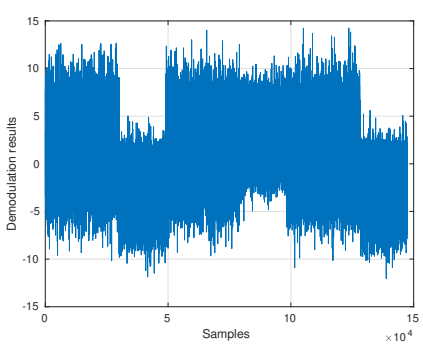

(a) Strategy 1

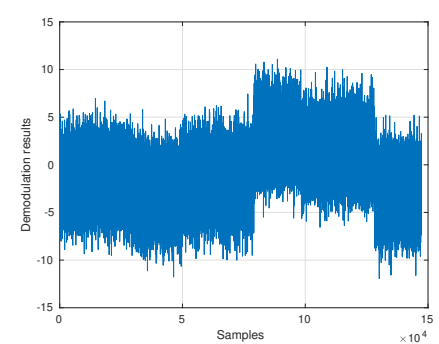

(b) Strategy 3

Fig. 11: Illustration of demodulation results for the three symbols based on different strategies.

spreading code of Galileo E1 OS signals is 4092:

$$
\begin{aligned}
& a_{1} T_{L C}<a_{2}\left(4092-T_{L C}\right) \\
& \Longrightarrow y=\frac{T_{L C}}{4092-T_{L C}}-\frac{a_{2}}{a_{1}}<0
\end{aligned}
$$

Fig. 12 shows the relation between $\frac{a_{2}}{a_{1}}$ and $T_{L C}$ to satisfy Eq. 21. If the attacker wants to maintain the same threshold as that for the aforementioned GPS signals, e.g., $\frac{a_{2}}{a_{1}}=1.2$, then $T_{L C}<2232$ chips.

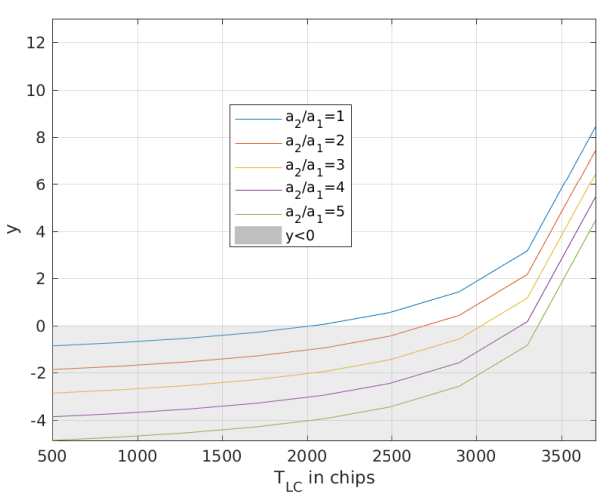

Fig. 12: Relation between $T_{L C}$ and the two parts signal power ratio $\frac{a_{2}}{a_{1}}$ for Galileo E1-B signals

\section{Consequences}

The maximum effect of the distance-decreasing attacks varies depending on the symbol length of different signals, as shown in Table. I. For unprotected signals, e.g., GPS L1 C/A, $T_{E D}$ and $T_{L C}$ are integer multiples of the spreading code length, hence the time gain is at the level of ms, which 
TABLE I: The effects of distance-decreasing attacks on different GNSS signals

\begin{tabular}{|c|c|c|c|c|c|c|c|c|}
\hline \multirow[b]{2}{*}{ Signals } & \multirow{2}{*}{$\begin{array}{c}\text { Non-protection signals } \\
\text { GPS L1 C/A } \\
\text { BeiDou B1I and B2I }\end{array}$} & \multirow{2}{*}{$\begin{array}{l}\text { NMA signals } \\
\text { Galileo E1 OS }\end{array}$} & \multicolumn{6}{|c|}{ Spreading-code encrypted signals } \\
\hline & & & $\begin{array}{l}\text { GPS L1 } \\
\text { P(Y) Code }\end{array}$ & $\begin{array}{l}\text { GPS } \\
\text { M-code }\end{array}$ & $\begin{array}{l}\text { BeiDou } \\
\text { B1Q }\end{array}$ & $\begin{array}{l}\text { BeiDou } \\
\text { B2Q }\end{array}$ & $\begin{array}{l}\text { Galileo } \\
\text { E1 PRS }\end{array}$ & $\begin{array}{l}\text { Galileo } \\
\text { E6 }\end{array}$ \\
\hline Target range & $\begin{array}{l}\text { Integer multiple of spreading } \\
\text { code length }\end{array}$ & $\begin{array}{l}\text { Fraction of one } \\
\text { spreading code } \\
\text { length }\end{array}$ & \multicolumn{6}{|c|}{ Fraction of one spreading code chip length } \\
\hline \multirow[b]{2}{*}{ Level of $T_{D D}$} & \multirow[b]{2}{*}{$\mathrm{ms}$} & \multirow[b]{2}{*}{$\mathrm{ns}$ to $\mathrm{ms}$} & \multicolumn{6}{|c|}{ Fraction of } \\
\hline & & & $2 \mu \mathrm{s}$ & $\begin{array}{l}195.5 \\
\mathrm{~ns}\end{array}$ & $\begin{array}{l}488.8 \\
\text { ns }\end{array}$ & $97.8 \mathrm{~ns}$ & $391 \mathrm{~ns}$ & $\begin{array}{l}195.5 \\
\text { ns }\end{array}$ \\
\hline $\begin{array}{l}\text { Shortened distance } \\
\text { (pseudorange mea- } \\
\text { surement) }\end{array}$ & $\geq 300 \mathrm{~km}$ & $\begin{array}{c}\text { meters to } \\
\text { hundreds of } \mathrm{km}\end{array}$ & \multicolumn{6}{|c|}{ meters to hundreds of $\mathrm{km}$} \\
\hline
\end{tabular}

consequently causes a shortening of the pseudorange at the order of $300 \mathrm{~km}$. For NMA signals, e.g., the Galileo E1B signals, both $T_{E D}$ and $T_{L C}$ are a fraction of the spreading code length, with $T_{L C}>T_{E D} ; T_{D D}$ can range from a single chip of the spreading code to a couple of thousand of chips. Therefore, the resultant decrease of pseudoranges for NMA signals can range from meters till hundreds of kilometers. For spreading-code encrypted signals, the chip length differs per different types of signals, e.g., $2 \mu$ s for the GPS P(Y) code signals and $391 \mathrm{~ns}$ for the Galileo E1 PRS signals; therefore, the DD attacker can affect pseudoranges at a level ranging from meters to hundreds of meters.

\section{COUnTERmeasures}

A distance-decreasing attacker tries to avoid having the ATX signal detected at the victim receiver (HRX) by resorting to different methods. In contrast, the GNSS receiver can use detection schemes for based on the understanding of the instantiation of DD attacks. In Eqs. 4, 13 and 18, first part of the ATX LC transmission, part of a bit, symbol, or chip, is the one that does not match the actual HTX transmission. When the honest receiver tries to determine the bit/symbol value, it integrates the signal energy within a period equal to the symbol length. For the LC approach 1, the ATX transmits dataless signals for $T_{L C}$, thus the demodulation result is noise until the beginning of the ARX-supported LC signal. This means, no significant negative contribution to the accumulation of one symbol energy. If the victim receiver checks closely the tracking results, as shown in the second plot of Fig. 9, it can identify the abnormal tracking results at the beginning part of each symbol. This could serve as evidence of a DD attack. Monitoring signal power would not be effective because the attacker does not need to transmit a high power signal for the second part of the LC signa.

For the second approach, the accumulated energy over $T_{L C}$ is almost zero because of the alternating $\{+C A$ code,$-C A$ code $\}$ for encoding data. Therefore, there is no negative effect on the bit value determination,as presented in the third plot of Fig. 9. Moreover, the attacker does not need to send increased power for the $T_{P S}$ part, as shown in Fig. 1c, to compensate the randomness of the pre-defined signal. However, if the HRX implements a detection scheme at the signal processing level, it can monitor for unusual rapid changes of correlation results, as an indication of a DD attack. Same as for the ATX LC apporach 1, monitoring for high signal powerwould not be effective.

For the ATX LC approach 3, the attacker-selected symbol value has $50 \%$ chance to be correct/wrong. Therefore, the power of the signal over $T_{P S}$, must be higher than that over $T_{L C}$, so that the energy accumulation over the entire bit/symbol provides a correct result. The increased power can be used as evidence of a DD attack, which the attacker can avoid by choosing $T_{L C}$ properly to satisfy Eq. 20 or 21 with knowledge of $\frac{a_{2}}{a_{1}}$. For spreading-code encrypted signals, the implementation of the attacker is at the sample level, with the strong assumption of good synchronization, which is very hardly to detect at a receiver.

\section{CONCLUSIONS}

We investigated distance-decreasing attacks for different GNSS signals, with and without cryptographic protection. We first explored how the attack components of early detection and late commit can be implemented for non-protected GNSS signals, such as the GPS L1 C/A code signals, an easier target compared to cryptographically protected signal. We also explored DD attacks on cryptographically protected signals, notably NMA and spreading-code encrypted signals. The implementation of DD attacks on spreading-code encrypted signals is hard because the attacker has very little information about the signal. If the attacker has good synchronization with the encrypted signal, it can implement DD attackss based on each sample of the secret code chip. However, it is difficult to achieve this synchronization; finding a pattern of secret spreading codes by analyzing a large volume of collected data could be a potential direction. Moreover, we evaluated the performance of the victim receiver while its signal has been manipulated by a distance-decreasing attacker. We showed how DD attacks affect tracking and demodulation results for the GPS L1 C/A and the Galileo E1B OS signals. The consequence of the attacks is shorted pseudorange measurements that vary from meters to hundreds of kilometers for different GNSS signals. We made a set of preliminary observations 
concerning countermeasures that seek to detect an ongoing DD attack.

In our upcoming work, we will evaluate the performance of a software receiver with Galileo raw signals. We will implement DD attacks on software defined radio and demonstrate the attack processes to see how it affects the receiver in different scenarios. We will develop protection schemes tailored to the DD attacks. Moreover, we will expand the discussion on the spreading-code encrypted GNSS signals, considering synchronization, methods used for early detection to have a high correct estimation rate and methods to reconstruct not easily detected late-commit signals.

\section{ACKNOWLEDGMENT}

This work has been partially supported by the Swedish Foundation for Strategic Research (SSF) SURPRISE project.

\section{REFERENCES}

[1] D. M. Akos, "Who's afraid of the spoofer? GPS/GNSS spoofing detection via automatic gain control (AGC)," Navigation, vol. 59, no. 4, pp. 281-290, 2012

[2] F. Bastide, D. Akos, C. Macabiau, and B. Roturier, "Automatic gain control (AGC) as an interference assessment tool," in ION GPS/GNSS 2003, 16th International Technical Meeting of the Satellite Division of The Institute of Navigation, 2003, pp. pp-2042.

[3] K. Borre, D. M. Akos, N. Bertelsen, P. Rinder, and S. H. Jensen, A software-defined GPS and Galileo receiver: a single-frequency approach. Springer Science \& Business Media, 2007.

[4] G. Caparra, S. Sturaro, N. Laurenti, C. Wullems, and R. T. Ioannides, "A novel navigation message authentication scheme for gnss open service," in ION GNSS, vol. 2016, 2016.

[5] J. Clulow, G. P. Hancke, M. G. Kuhn, and T. Moore, "So near and yet so far: Distance-bounding attacks in wireless networks," in European Workshop on Security in Ad-hoc and Sensor Networks. Springer, 2006, pp. 83-97.

[6] I. Fernández-Hernández, V. Rijmen, G. Seco-Granados, J. Simon, I. Rodríguez, and J. D. Calle, "A navigation message authentication proposal for the galileo open service," Navigation: Journal of the Institute of Navigation, vol. 63, no. 1, pp. 85-102, 2016.

[7] G. Hein, F. Kneissl, J.-A. Avila-Rodriguez, and S. Wallner, "Authenticating gnss: proofs against spoofs, part 2," Inside GNSS, vol. 2, no. 5 , pp. 58-63, 2007.

[8] T. E. Humphreys, "Detection strategy for cryptographic GNSS antispoofing," IEEE Transactions on Aerospace and Electronic Systems, vol. 49, no. 2, pp. 1073-1090, 2013.

[9] T. E. Humphreys, J. A. Bhatti, D. Shepard, and K. Wesson, "The Texas spoofing test battery: Toward a standard for evaluating GPS signal authentication techniques," in Radionavigation Laboratory Conference Proceedings, 2012.

[10] T. E. Humphreys, B. M. Ledvina, M. L. Psiaki, B. W. O’Hanlon, and P. M. Kintner Jr, "Assessing the Spoofing Threat," GPS World, vol. 20, no. 1 , pp. 28-38, 2009.

[11] D. Marnach, S. Mauw, M. Martins, and C. Harpes, "Detecting Meaconing Attacks by Analysing the Clock Bias of GNSS Receivers," Artificial Satellites, vol. 48, no. 2, pp. 63-83, 2013.

[12] P. Y. Montgomery, T. E. Humphreys, and B. M. Ledvina, "Receiverautonomous spoofing detection: Experimental results of a multi-antenna receiver defense against a portable civil GPS spoofer,' in Proceedings of the ION International Technical Meeting, 2009, pp. 124-130.

[13] P. Papadimitratos and A. Jovanovic, "GNSS-based positioning: Attacks and Countermeasures," in Military Communications Conference, 2008. MILCOM 2008. IEEE. IEEE, 2008, pp. 1-7.

[14] — "Method to secure GNSS based locations in a device having GNSS receiver," Apr. 17 2012, US Patent 8,159,391.

[15] M. Poturalski, M. Flury, P. Papadimitratos, J.-P. Hubaux, and J.-Y. Le Boudec, "On secure and precise ir-uwb ranging," IEEE Transactions on Wireless Communications, vol. 11, no. 3, pp. 1087-1099, 2012.
[16] M. L. Psiaki, B. W. O’hanlon, S. P. Powell, J. A. Bhatti, K. D. Wesson, and T. E. Humphreys, "GNSS spoofing detection using two-antenna differential carrier phase," 2014.

[17] The European Union, "Galileo Open Service Signal-In-Space Interface Control Document (OS SIS ICD), Issue 1.2," 2015.

[18] P. Walker, V. Rijmen, I. Fernandez-Hernandez, G. Seco-Granados, J. Simón, J. Calle, and O. Pozzobon, "Galileo open service authentication: a complete service design and provision analysis," in Proceedings of the 28th International Technical Meeting of the Satellite Division of The Institute of Navigation (ION GNSS+ 2015), 2015, pp. 3383-3396.

[19] K. Wesson, M. Rothlisberger, and T. Humphreys, "Practical cryptographic civil GPS signal authentication," Navigation, vol. 59, no. 3, pp. 177-193, 2012

[20] K. Zhang and P. Papadimitratos, "GNSS receiver tracking performance analysis under distance-decreasing attacks," in 2015 International Conference on Location and GNSS (ICL-GNSS). IEEE, 2015, pp. 1-6. 